\title{
On the multiplicativity of quantum cat maps
}

\author{
Francesco Mezzadri周团 \\ American Institute of Mathematics, 360 Portage Ave, Palo Alto, CA 94306, USA \\ E-mail: f.mezzadri@bris.ac.uk \\ 8 April 2002
}

\begin{abstract}
The quantum mechanical propagators of the linear automorphisms of the two-torus (cat maps) determine a projective unitary representation of the theta group $\Gamma_{\theta} \subset \mathrm{SL}(2, \mathbb{Z})$. We prove that there exists an appropriate choice of phases in the propagators that defines a proper representation of $\Gamma_{\theta}$. We also give explicit formulae for the propagators in this representation.
\end{abstract}

Mathematics Subject Classification: 20C35, 20H05, 81Q50

\section{Introduction}

The quantization of the linear automorphisms of the two-torus (cat maps) was first developed by Hannay and Berry [12], who were able to determine the quantum propagators for the subgroup of $\mathrm{SL}(2, \mathbb{Z})$ defined by

$$
\Gamma_{\theta}=\left\{A=\left(\begin{array}{ll}
a & b \\
c & d
\end{array}\right) \in \mathrm{SL}(2, \mathbb{Z}) \mid a b \equiv c d \equiv 0 \bmod 2\right\} .
$$

This set of matrices is often known as the theta group. It turns out that it is possible to associate to every element of $\mathrm{SL}(2, \mathbb{Z})$ a propagator 8 , 4 , or quantum map, which is a unitary operator acting on a finite dimensional Hilbert space. However, the multiplication among propagators of different cat maps can be defined only if the quantization is restricted to certain subgroups of $\mathrm{SL}(2, \mathbb{Z})$, which depend on the periodicity conditions imposed on the quantum wavefunction (see, e.g. [8, 4, 7]). It turns out that the theta group is the largest of these subgroups.

An exact form of Egorov's theorem characterizes the quantum cat maps, which, as a consequence, are multiplicative up to a phase factor, i.e.

$$
U_{N}(A B)=\mathrm{e}^{2 \pi \mathrm{i} \zeta(A, B)} U_{N}(A) U_{N}(B), \quad A, B \in \Gamma \subset \mathrm{SL}(2, \mathbb{Z}),
$$

$\dagger$ Supported by a Royal Society Dorothy Hodgkin Fellowship.

$\ddagger$ Permanent address: Department of Mathematics, University of Bristol, University Walk, Bristol BS8 1TW, UK. 
where $U_{N}(A)$ denotes the propagator, $\Gamma$ is a subgroup of $\mathrm{SL}(2, \mathbb{Z})$ and $N$ is the dimension of the Hilbert space in which $U_{N}(A)$ acts. In other words, the quantization determines a projective representation of $\Gamma$, which is sometimes referred to as Weil's representation.

In their original paper, Hannay and Berry conjectured that the quantization of the cat maps is multiplicative in the theta group, i.e. it defines a proper representation. The importance of this property was emphasized by Kurlberg and Rudnick [10], who proved that if we restrict to the congruence subgroup defined by $A \equiv 1_{2} \bmod 2$ for $N$ odd and $A \equiv 1_{2} \bmod 4$ for $N$ even, where $1_{2}$ denotes the $2 \times 2$ identity matrix, then it is possible to choose the propagators so that the quantization is multiplicative. The purpose of this paper is to prove that there exists a choice of phases of the propagators $U_{N}(A)$ that determines a proper representation of the whole theta group. We also give an explicit expression for these phases.

The multiplicativity of the propagators is strictly related to the existence of a set of unitary operators - known as Hecke operators in analogy with a similar phenomenon in the theory of modular surfaces - that commute with the map and among themselves. Indeed, it can be shown that multiplicativity implies the existence of these symmetries. Most of the mathematical properties of the quantum cat maps, like the degeneracy in their spectra, are due to the Hecke operators. Kurlberg and Rudnick [10] proved that the set of simultaneous eigenfunctions of a quantum cat map and of its Hecke symmetries (the Hecke eigenfunctions) become equidistributed in the semiclassical limit with respect to Liouville measure. They also obtained rigorous results on the value distribution and extreme values of a particular class of Hecke eigenfunctions [11. Furthermore, the Hecke operators are responsible for spectral statistics of a significant class of perturbations of the quantum cat maps that, although being consistent with distributions of eigenvalues of random matrices, do not belong to the universality classes expected from the symmetries of the classical dynamics [6].

The outline of the article is as follows. In section 2 the quantum cat maps are introduced and the main results are presented. The implications of the quantization defined in this paper on the structure of the Hecke operators are described in section 3. Section 4 is devoted to the proof of multiplicativity for the theta group. In sections 5 and 6 we determine a choice of quantum propagators that defines a proper representation of the theta group. This will also give an alternative proof of multiplicativity.

\section{The quantum cat maps}

The cat maps are the linear automorphisms of the two-torus $\mathbb{T}^{2}=\mathbb{R}^{2} / \mathbb{Z}^{2}$. Their dynamics may be represented by the action of elements of the modular group $\operatorname{SL}(2, \mathbb{Z})$ modulo one. In other words, we consider the symplectic map

$$
\boldsymbol{z} \mapsto A \cdot \boldsymbol{z}, \quad \boldsymbol{z}=\left(\begin{array}{c}
q \\
p
\end{array}\right) \in \mathbb{T}^{2}, \quad A \in \mathrm{SL}(2, \mathbb{Z}) .
$$

The torus plays the role of phase space, therefore the coordinates $q$ and $p$ are taken to represent the position variable and its conjugate momentum. 
The action of $A$ on $\mathbb{T}^{2}$ can be interpreted in terms of the discrete time evolution of a dynamical system, thus the corresponding quantum dynamics is determined by a unitary operator $U_{N}(A)$. When $|\operatorname{Tr}(A)|>2$, the dynamics generated by the classical map is hyperbolic. Since the phase space is compact, the Hilbert space $\mathcal{H}_{N}$ on which $U_{N}(A)$ acts is finite dimensional and may be identified with $\mathrm{L}^{2}(\mathbb{Z} / N \mathbb{Z})$, where $\mathbb{Z} / N \mathbb{Z}$ is the ring of congruence classes modulo $N$. The dimension of $\mathcal{H}_{N}$ and Planck's constant are related via the condition $2 \pi \hbar=1 / N$. (We refer the reader to appendix $\mathrm{B}$ for more details on the quantization of maps on the torus.)

There are many standard ways of mapping classical observables $f \in C^{\infty}\left(\mathbb{T}^{2}\right)$ into operators $\mathrm{Op}_{N}(f)$ acting on $\mathcal{H}_{N}$. However, elements of $\mathrm{SL}(2, \mathbb{Z})$ cannot be quantized using these techniques because, although they are symplectic, they cannot be interpreted as a one-time flow of a Hamiltonian on $\mathbb{T}^{2}[7]$. Therefore, they need an ad hoc quantization procedure. Since we are considering linear systems, it is natural to require that the quantum and classical evolution commute, or, more precisely, that an exact form of Egorov's theorem holds:

$$
U_{N}(A)^{-1} \mathrm{Op}_{N}(f) U_{N}(A)=\mathrm{Op}_{N}(f \circ A), \quad f \in C^{\infty}\left(\mathbb{T}^{2}\right) .
$$

An alternative way of understanding (2.1) is the following. The operator $\operatorname{Op}_{N}(f)$ can be expressed as a linear combination of elements of an irreducible representation $\left(\tau, \mathcal{H}_{N}\right)$ with a given central character $\chi$ of a finite Heisenberg group $H$ (see, e.g. [10]). Because of the Stone-von Neumann theorem, there exists a unique isomorphism class of irreducible representations of $H$ with central character $\chi$. Then, $A \in \mathrm{SL}(2, \mathbb{Z})$ can be projected into an automorphism of $H$ that fixes the centre, and thus the map $\tau_{A}(h)=\tau\left({ }^{A} h\right)$, with $h \in H$, defines an irreducible representation $\left(\tau_{A}, \mathcal{H}_{N}\right)$ as long as the two central characters are the same, i.e. $\chi^{A}=\chi$. These two representations must be isomorphic. The intertwining operator $U_{N}(A): \mathcal{H}_{N} \rightarrow \mathcal{H}_{N}$ from $\left(\tau, \mathcal{H}_{N}\right)$ to $\left(\tau_{A}, \mathcal{H}_{N}\right)$ is known as quantum propagator of $A$. Because of Schur's lemma, $U_{N}(A)$ is unique up to a phase factor. Many approaches have been developed to determine $U_{N}(A)$ [12, 8, 6, 3, 19, 10], which, because of (2.1), are all equivalent up to phase factors.

The topology of the torus constrains the quantum wavefunction to be periodic up to a phase factor in both the position and momentum representations, i.e.

$$
\begin{aligned}
& \psi\left(q+m_{1}\right)=\mathrm{e}\left(m_{1} \varphi_{2}\right) \psi(q) \\
& \hat{\psi}\left(p+m_{2}\right)=\mathrm{e}\left(-m_{2} \varphi_{1}\right) \hat{\psi}(p), \quad \boldsymbol{\varphi}=\left(\begin{array}{c}
\varphi_{1} \\
\varphi_{2}
\end{array}\right) \in \mathbb{T}^{2}, \quad \boldsymbol{m}=\left(\begin{array}{c}
m_{1} \\
m_{2}
\end{array}\right) \in \mathbb{Z}^{2},
\end{aligned}
$$

where $\mathrm{e}(x):=\mathrm{e}^{2 \pi \mathrm{i} x}$ and

$$
\hat{\psi}(p)=\frac{1}{\sqrt{2 \pi \hbar}} \int_{-\infty}^{+\infty} \psi(q) \text { e }\left(-\frac{q p}{2 \pi \hbar}\right) \mathrm{d} q .
$$

Elements of $\operatorname{SL}(2, \mathbb{Z})$ can be quantized only when $\varphi_{1}$ and $\varphi_{2}$ are rational numbers and, given a $\varphi \in \mathbb{Q}^{2} / \mathbb{Z}^{2}$, the quantization is restricted to a certain subgroup of $\operatorname{SL}(2, \mathbb{Z})$ (see, e.g. [8, [4, 3, 团). When $\varphi=(0,0)$, then the maps that can be quantized belong to $\Gamma_{\theta}$. These restrictions are due to the constraint that the central characters $\chi$ and 
$\chi^{A}$ of the two irreducible representations $\left(\tau, \mathcal{H}_{N}\right)$ and $\left(\tau_{A}, \mathcal{H}_{N}\right)$ must be the same. The theta group has index three in $\mathrm{SL}(2, \mathbb{Z})$ and is the largest subgroup of $\mathrm{SL}(2, \mathbb{Z})$ that can be quantized once the periodicity conditions have been fixed, in the sense that when $\boldsymbol{\varphi} \neq(0,0)$ the quantization is restricted to groups of higher index in $\mathrm{SL}(2, \mathbb{Z})$. In this article we shall consider only the case when $\boldsymbol{\varphi}=(0,0)$, i.e. the wavefunction is exactly periodic in both position and momentum representations.

It is easy to see that Egorov's theorem determines a projective representation of $\Gamma_{\theta}$. The aim of this paper is to prove that it is possible to define a proper representation of $\Gamma_{\theta}$. We have the following result.

Theorem 2.1. For any positive integer $N$, there exists a choice of phases in the definition of the propagators $U_{N}(A)$ such that

$$
U_{N}(A B)=U_{N}(A) U_{N}(B), \quad A, B \in \Gamma_{\theta} .
$$

A choice of propagators that realizes (2.9) is the following. If

$$
S^{ \pm}=\left(\begin{array}{cc}
0 & \mp 1 \\
\pm 1 & 0
\end{array}\right)
$$

then the propagator is the discrete Fourier transform:

$$
\left[U_{N}\left(S^{ \pm}\right) \Phi\right](Q):=\frac{1}{\sqrt{N}} \sum_{Q^{\prime} \bmod N} \mathrm{e}\left(\frac{ \pm Q Q^{\prime}}{N}\right) \Phi\left(Q^{\prime}\right), \quad \Phi \in \mathcal{H}_{N}
$$

The parity matrix

$$
P=\left(\begin{array}{cc}
-1 & 0 \\
0 & -1
\end{array}\right)
$$

has the obvious quantization

$$
\left[U_{N}(P) \Phi\right](Q):=\Phi(-Q), \quad \Phi \in \mathcal{H}_{N} .
$$

If $b=0$, i.e.

$$
T_{m}^{ \pm}=\left(\begin{array}{cc} 
\pm 1 & 0 \\
m & \pm 1
\end{array}\right), \quad m \equiv 0 \bmod 2
$$

then we have

$$
\left[U_{N}\left(T_{m}^{ \pm}\right) \Phi\right](Q):=\mathrm{e}\left(\frac{ \pm m Q^{2}}{2 N}\right) \Phi( \pm Q), \quad \Phi \in \mathcal{H}_{N} .
$$

Similarly, if $a=0$, i.e.

$$
W_{w}^{ \pm}=\left(\begin{array}{cc}
0 & \pm 1 \\
\mp 1 & w
\end{array}\right), \quad w \equiv 0 \bmod 2,
$$

then we set

$$
\left[U_{N}\left(W_{w}^{ \pm}\right) \Phi\right](Q):=\frac{1}{\sqrt{N}} \sum_{Q^{\prime} \bmod N} \mathrm{e}\left(\frac{ \pm 1}{2 N}\left(w Q^{2}-2 Q Q^{\prime}\right)\right) \Phi\left(Q^{\prime}\right), \quad \Phi \in \mathcal{H}_{N}
$$


In all the other cases we define

$$
\begin{aligned}
{\left[U_{N}(A) \Phi\right](Q):=} & \frac{h(a, b)}{\sqrt{N_{b}}} \sum_{Q^{\prime} \bmod N} G\left(\alpha, \beta, \gamma\left(Q, Q^{\prime}\right)\right) \\
& \times \mathrm{e}\left(\frac{1}{2 N b}\left(d Q^{2}-2 Q Q^{\prime}+a Q^{\prime 2}\right)\right) \Phi\left(Q^{\prime}\right), \quad \Phi \in \mathcal{H}_{N},
\end{aligned}
$$

where $h(a, b)$ and $G(\alpha, \beta, \gamma)$ are defined in equations (2.4) and (2.5) respectively.

Formula $(2.38)$ needs a few words of explanation. Firstly, we have

$$
h(a, b):= \begin{cases}\left(\frac{|a|}{|b|}\right) \mathrm{e}(\operatorname{sign}(a b)(|b|-1) / 8) & \text { if } a \text { is even } \\ \left(\frac{|b|}{|a|}\right) \mathrm{e}(-\operatorname{sign}(a b)|a| / 8) & \text { if } a \text { is odd }\end{cases}
$$

where $\left(\frac{p}{q}\right)$ is the Jacobi symbol (see appendix A for the definition and properties of the Jacobi symbol) and $\operatorname{sign}(x)$ is the sign function

$$
\operatorname{sign}(x):= \begin{cases}1 & \text { if } x>0 \\ -1 & \text { if } x<0 \\ 0 & \text { if } x=0 .\end{cases}
$$

The function $h(a, b)$ is a phase factor which distinguishes definition (2.38) from the quantization that Hannay and Berry introduced in their original article [12. More precisely, Hannay and Berry's propagator is $U_{N}^{\mathrm{HB}}(A)=\sqrt{i} U_{N}(A) / h(a, b)$.

The term $G\left(\alpha, \beta, \gamma\left(Q, Q^{\prime}\right)\right)$ is a normalized Gauss sum:

$$
G\left(\alpha, \beta, \gamma\left(Q, Q^{\prime}\right)\right):=\frac{1}{\sqrt{|\beta|}} \sum_{k \bmod |\beta|} \mathrm{e}\left(\frac{1}{2 \beta}\left(\alpha k^{2}+\gamma\left(Q, Q^{\prime}\right) k\right)\right)
$$

where $\alpha:=N_{b} a, \beta:=b^{\prime}, \gamma:=2\left(a Q^{\prime}-Q\right) /(b, N)$ and $(b, N)$ is the greatest common divisor of $b$ and $N$, which we shall always take to be positive. In the previous definitions, in 2.38 and in what follows we use the notation

$$
\begin{array}{ll}
N_{a}:=N /(a, N), & a^{\prime}:=a /(a, N), \\
N_{b}:=N /(b, N), & b^{\prime}:=b /(b, N), \\
N_{d}:=N /(d, N), & d^{\prime}:=d /(d, N), \\
N_{a b}:=N /((a, N)(b, N)), & N_{b d}:=N /((b, N)(d, N)) .
\end{array}
$$


The sum (2.5) is different from zero only if $\alpha$ and $\beta$ are coprime integers, $\gamma$ is also an integer and $\alpha \beta+\gamma$ is even. $G(\alpha, \beta, \gamma)$ can be explicitly computed:

$$
G(\alpha, \beta, \gamma)=\left\{\begin{array}{l}
\left(\frac{|\alpha|}{|\beta|}\right) \mathrm{e}(-\operatorname{sign}(\alpha \beta)(|\beta|-1) / 8) \mathrm{e}\left(-\frac{\alpha(\alpha \backslash|\beta|)^{2}}{2 \beta}\left(\frac{\gamma}{2}\right)^{2}\right) \\
\text { if } \alpha \text { is even, } \beta \text { odd and } \gamma \text { even, } \\
\left(\frac{|\beta|}{|\alpha|}\right) \mathrm{e}(\operatorname{sign}(\alpha \beta)|\alpha| / 8) \mathrm{e}\left(-\frac{\alpha(\alpha \backslash \beta \mid)^{2}}{2 \beta}\left(\frac{\gamma}{2}\right)^{2}\right) \\
\text { if } \alpha \text { is odd, } \beta \text { even and } \gamma \text { even, } \\
\left(\frac{|\alpha|}{|\beta|}\right) \mathrm{e}(-\operatorname{sign}(\alpha \beta)(|\beta|-1) / 8) \mathrm{e}\left(-2 \alpha(4 \alpha \backslash|\beta|)^{2} \gamma^{2} / \beta\right) \\
\text { if } \alpha \text { is odd, } \beta \text { odd and } \gamma \text { odd. }
\end{array}\right.
$$

Here $(p \backslash q)$ denotes the inverse integer of $p$ modulo $q$, where $p$ and $q$ are mutually prime, i.e. the only integer modulo $q$ such that

$$
p(p \backslash q) \equiv 1 \bmod q .
$$

Moreover, the Euler-Fermat theorem gives

$$
(p \backslash q) \equiv p^{\phi(q)-1} \bmod q,
$$

where $\phi(q)$ is Euler's function, which is defined as the number of integers less than and mutually prime to $q$. (For a detailed explanation of (2.5) and (2.6) we refer the reader to [12].)

It was pointed out by Hannay and Berry [12] that the Gauss sum (2.5) is invariant if

$$
N_{b} a \rightarrow N_{b} d, \quad \frac{2}{(b, N)}\left(a Q^{\prime}-Q\right) \rightarrow \frac{2}{(b, N)}\left(d Q-Q^{\prime}\right) .
$$

The reason is quite simple: if $f(Q)$ is a function defined on $\mathbb{Z} / m \mathbb{Z}$, then the sum $\sum_{Q \bmod m} f(Q)$ is invariant if $Q \rightarrow l Q$, where $(l, m)=1$. The substitutions (2.7) are equivalent to replacing $k$ by $-d k$ in (2.5). Similarly, we have the equality

$$
h(a, b)=h(d, b)
$$

which is proven in appendix $\mathrm{C}$.

It is straightforward to check that the operators $(2.3 d),(2.3 b),(2.3 \mathrm{~d}$ ) and $(2.3 \mathrm{~d})$ satisfy (2.1); it was shown by Knabe [8] and Degli Esposti [4] that the propagator introduced by Hannay and Berry [12], and therefore also definition (2.38), obeys an exact form of Egorov's theorem. Thus, the quantization procedure that we gave defines a projective representation of $\Gamma_{\theta}$. In the following sections we will prove that it is proper representation. In section 3 we will show that the definitions of the propagators $(2.3)$ depend only on the reduction of $A$ modulo $4 N$. Therefore, since the projection

$$
\pi: \mathrm{SL}(2, \mathbb{Z}) \rightarrow \mathrm{SL}(2, \mathbb{Z} / 4 N \mathbb{Z})
$$

is surjective (see, e.g. [17]), formulae (2.3) also define a representation of the group

$$
\Gamma_{\theta}(4 N)=\left\{A=\left(\begin{array}{ll}
a & b \\
c & d
\end{array}\right) \in \mathrm{SL}(2, \mathbb{Z} / 4 N \mathbb{Z}) \mid a b \equiv c d \equiv 0 \bmod 2\right\} .
$$


There exist partial results in the direction of the first part of theorem 2.1. The first goes back to Schur [18], who proved that when $p$ is an odd prime, any projective representation of $\mathrm{SL}(2, \mathbb{Z} / p \mathbb{Z})$ can be modified to give a representation. An analogous result was obtained in $[15, \mathbb{1}$. More generally, the same property holds for $\operatorname{SL}(2, \mathbb{Z} / m \mathbb{Z})$ when $m \not \equiv 0 \bmod 4$ [14, 2]. Kurlberg and Rudnick [10] proved that there exists a choice of propagators that defines a representation of the congruence subgroup

$$
\Gamma(4,2 N)=\left\{A \in \mathrm{SL}(2, \mathbb{Z} / 2 N \mathbb{Z}) \mid\left\{\begin{array}{ll}
A \equiv 1_{2} \bmod 2 & \text { for } N \text { odd } \\
A \equiv 1_{2} \bmod 4 & \text { for } N \text { even }
\end{array}\right\} .\right.
$$

Similar results can be found in [9, 5].

\section{The Hecke operators}

The quantum cat maps are characterized by the existence of a group of commutative unitary symmetries known as Hecke operators. As mentioned in the introduction, these symmetries are responsible of most of the arithmetical properties of the quantum cat maps. Since theorem 2.1 has important consequence on the structure of such operators, we briefly introduce them and describe how they are related to the propagators defined in formulae (2.3). The proof of theorem 2.1 will be presented in the following sections.

If two cat maps are equivalent modulo $2 N$, then their quantum propagators differ by a phase factor. This property was already discovered by Hannay and Berry [12] and is a direct consequence of formulae (2.6). Kurlberg and Rudnick's [10] quantization is a map

$$
\rho: \mathrm{SL}(2, \mathbb{Z} / 2 N \mathbb{Z}) \rightarrow \mathrm{U}(N), \quad A \mapsto U_{N}^{\mathrm{KR}}(A) .
$$

So by construction, if $A \equiv B \bmod 2 N$ then $U_{N}^{\mathrm{KR}}(A)=U_{N}^{\mathrm{KR}}(B)$. Thus, multiplicativity implies the existence of Hecke operators. In fact, suppose that $A B \equiv B A \bmod 2 N$, then we have

$$
U_{N}^{\mathrm{KR}}(A B)=U_{N}^{\mathrm{KR}}(A) U_{N}^{\mathrm{KR}}(B)=U_{N}^{\mathrm{KR}}(B) U_{N}^{\mathrm{KR}}(A)=U_{N}^{\mathrm{KR}}(B A),
$$

i.e. $U_{N}^{\mathrm{KR}}(B)$ is a symmetry of $U_{N}^{\mathrm{KR}}(A)$, even though $A$ and $B$ do not commute exactly.

Kurlberg and Rudnick [10] proved that the map (3.1) can be defined in such a way that $\rho$ is a proper representation when restricted to the congruence subgroup $\Gamma(4,2 N)$. They also proved that given $A \in \Gamma(4,2 N)$ the number of elements of $\Gamma(4,2 N)$ that commute with $A$ and among themselves is of order $N$.

We conclude by showing that the propagators defined in formulae (2.3) depend only on the reduction of $A$ modulo $4 N$ and therefore they define a proper representation of $\Gamma_{\theta}(4 N)$. As a consequence, in this case the Hecke symmetries of a quantum cat map $U_{N}(A)$ are those $U_{N}(B)$ such that

$$
A B \equiv B A \bmod 4 N \text {. }
$$


The order of the group of Hecke symmetries of a given $U_{N}(A)$ is of order $N$ also in this case. This follows directly from Kurlberg and Rudnick's result, which implies that the number of equivalence classes of matrices modulo $4 N$ in

$$
\Gamma(4)=\left\{A \in \mathrm{SL}(2, \mathbb{Z}) \mid A \equiv 1_{2} \bmod 4\right\}
$$

that commute modulo $4 N$ among themselves and with a given $A$ is of order $N$. Since $\Gamma(4)$ is of finite order in $\Gamma_{\theta}$, the same statement holds in $\Gamma_{\theta}$. It is worth noting that the reduction modulo $4 N$ in this respect is essential, because the only matrices that commute exactly with $A \in \mathrm{SL}(2, \mathbb{Z})$ are $P$ and the powers of the primitive matrix of $A$ [8.

It also is important to point out that if there exists a choice of phases in the definition of the propagators such that

$$
U_{N}(A) U_{N}(B)=U_{N}(B) U_{N}(A)
$$

then $U_{N}(A)$ and $U_{N}(B)$ commute for any choice; this is not the case with the multiplicativity property $(2.2)$.

Finally, we have the following.

Corollary 3.1. The propagators $U_{N}(A)$ defined in formulae (2.3) depend only on the reduction of $A$ modulo $4 N$.

Proof. It is trivial to see that the propagators $(2.3 \mathrm{~d})$ and $(2.3 \mathrm{~d})$ are invariant if $m \mapsto m+4 N k$ and $w \mapsto w+4 N k^{\prime}$. Now, suppose that $A \equiv B \bmod 4 N$ and $U_{N}(A)$ and $U_{N}(B)$ are of the form $(2.3 \theta)$. We have

$$
A=B+4 N M=B\left(1_{2}+4 N B^{-1} M\right),
$$

where $M$ is a matrix with integer entries. The propagators of both sides of (3.2) are

$$
U_{N}(A)=U_{N}(B C)=U_{N}(B) U_{N}(C),
$$

where $C=1_{2}+4 N B^{-1} M$. We know that

$$
\left[U_{N}(C) \Phi\right](Q)=\mathrm{e}(\mu) \Phi(Q), \quad \Phi \in \mathcal{H}_{N}
$$

The phase factor $\mathrm{e}(\mu)$ can be determined directly from (2.3e) and (2.4):

$\mathrm{e}(\mu)=h(a, b)\left(\frac{|b| / N}{|a|}\right) \mathrm{e}(\operatorname{sign}(a b)|a| / 8)=\left(\frac{N}{|a|}\right), \quad C=\left(\begin{array}{ll}a & b \\ c & d\end{array}\right)$.

Now, since $|a|= \pm 1 \bmod 4 N$, from property (A.2) of the Jacobi symbol we have

$$
\left(\frac{N}{|a|}\right)=1 \text {. }
$$

$\S A_{0} \in \mathrm{SL}(2, \mathbb{Z})$ is primitive if $A_{0}=A^{k}, A \in \mathrm{SL}(2, \mathbb{Z})$, implies $k=1$. 
More generally, using (2.6) one can show that if

$$
A \equiv\left(\begin{array}{cc}
(a \backslash 4 N) & 0 \\
0 & a
\end{array}\right) \bmod 4 N, \quad(a, 4 N)=1,
$$

then

$$
\left[U_{N}(A) \Phi\right](Q)=\left(\frac{N}{|a|}\right) \Phi(a Q), \quad \Phi \in \mathcal{H}_{N} .
$$

Corollary 3.1 then follows immediately. It is also important to point out that if $A \equiv B \bmod 2 N$, then

$$
U_{N}(A)=\left(\frac{N}{|a|}\right) U_{N}(B)
$$

However, since $|a|= \pm 1 \bmod 2 N$, the Jacobi symbol $\left(\frac{N}{|a|}\right)$ is not necessarily one.

\section{Relations and multiplicativity for $\Gamma_{\theta}$}

Given an abstract group $\Gamma$, it is always possible to choose a subset

$$
G=\left\{g_{1}, g_{2}, \ldots, g_{n}, \ldots\right\}, \quad G \subset \Gamma
$$

such that each element of $\Gamma$ can be written as product, or string, of a finite number of $g_{i}$, i.e. $\forall h \in \Gamma, h=a_{1} a_{2} \cdots a_{r}$, where $a_{j}=g_{i}^{\epsilon}$ and $\epsilon= \pm 1$. We call $G$ a set of generators. The cardinality of $G$ may be finite or infinite; the groups that we are concerned with are all finitely generated.

It is clear that if we choose appropriately a set of generators of $\Gamma_{\theta}$ and then we understand how their propagators behave, we may be able to make some progress on the multiplicativity properties of arbitrary elements of $\Gamma_{\theta}$. Indeed, knowing a set of generators, say $G=\left\{g_{1}, g_{2}, \ldots, g_{n}\right\}$, one might be tempted to think that if the $U_{N}\left(g_{i}\right)$ obey (2.1) and we define

$$
U_{N}(A)=U_{N}\left(a_{1} \cdots a_{k}\right)=U_{N}\left(a_{1}\right) \cdots U_{N}\left(a_{k}\right), \quad a_{j}=g_{i}^{\epsilon}, \quad \epsilon= \pm 1,
$$

then multiplicativity would be automatic. This would be the case if $\Gamma_{\theta}$ were a free group, i.e. if no relations existed among the generators or, in other words, if each element of $\Gamma_{\theta}$ could be written in a unique way as a finite product $a_{1} \cdots a_{k}$ 团, each $a_{j}$ being some $g_{i}^{\epsilon}$, where $\epsilon= \pm 1$. However, in general a given $A \in \Gamma_{\theta}$ can be written in many different ways as product of generators and one must check that such relations hold among the $U_{N}\left(g_{i}\right)$ too.

The main result of this section is the following.

$\dagger$ More precisely, this statement is true only for reduced words, i.e. words where no pair $a_{j} a_{j+1}$, $j=1, \ldots, k-1$, is of the form $g_{i}^{\epsilon} g_{i}^{-\epsilon}$. 
Theorem 4.1. The group $\Gamma_{\theta}$ is generated by the set of matrices $G=\left\{S^{+}, P, T_{2}^{+}\right\}$, where

$$
S^{+}=\left(\begin{array}{cc}
0 & -1 \\
1 & 0
\end{array}\right), \quad P=\left(\begin{array}{cc}
-1 & 0 \\
0 & -1
\end{array}\right), \quad T_{2}^{+}=\left(\begin{array}{ll}
1 & 0 \\
2 & 1
\end{array}\right) .
$$

The only relations among $S^{+}, P, T_{2}^{+}$are

$$
P^{2}=1_{2}, \quad S^{+2} P=1_{2}, \quad S^{+} P=P S^{+}, \quad T_{2}^{+} P=P T_{2}^{+} .
$$

Furthermore, if $U_{N}\left(S^{+}\right), U_{N}(P)$ and $U_{N}\left(T_{2}^{+}\right)$are given by (2.3a), (2.38) and 2.3 , then the following relations hold:

$$
\begin{aligned}
& U_{N}(P)^{2}=1_{N}, \\
& U_{N}\left(S^{+}\right)^{2} U_{N}(P)=1_{N}, \\
& U_{N}\left(S^{+}\right) U_{N}(P)=U_{N}(P) U_{N}\left(S^{+}\right), \\
& U_{N}\left(T_{2}^{+}\right) U_{N}(P)=U_{N}(P) U_{N}\left(T_{2}^{+}\right) .
\end{aligned}
$$

This theorem automatically gives $U_{N}(A B)=U_{N}(A) U_{N}(B)$ once $U_{N}(A)$ is defined according to (4.1). However, $U_{N}(A)$ is given implicitly as product of propagators of generators and this may be inconvenient in applications. We shall address this issue in sections 5 and 6 .

The statement that $G=\left\{S^{+}, P, T_{2}^{+}\right\}$is a set of generators of $\Gamma_{\theta}$ with relations (4.3) is a direct consequence of well known results in the theory of modular forms (see, e.g. [16]). However, since it is fundamental to our work, we shall provide a complete proof. The last part of the theorem can be easily checked by direct multiplication.

We begin by proving that $S^{+}$and $T_{2}^{+}$generate $\Gamma_{\theta}$. Then, since $S^{+^{2}}=P, G$ is also a set of generators.

Lemma 4.2. The matrices $T_{2}^{+}$and $S^{+}$are a set of generators of $\Gamma_{\theta}$.

Proof. The proof consists of showing that by successive multiplications from the right by $T_{ \pm 2}^{+}$and $S^{ \pm}$an arbitrary matrix $A \in \Gamma_{\theta}$ can be reduced to either $T_{ \pm 2}^{+}$or $S^{ \pm}$.

Let us consider first the two cases when $b=0$ and $b= \pm 1$. Firstly, suppose that $b=0$, i.e. $A=T_{m}^{ \pm}$but $m \neq \pm 2$. If $A=T_{m}^{-}$then by applying $P=S^{+^{2}}$ we obtain $T_{-m}^{+}$. Clearly, $A$ can be reduced to $T_{ \pm 2}^{+}$by repeated multiplications from the right by $T_{ \pm 2}$. If, instead, $b= \pm 1$, repeated applications of $T_{ \pm 2}^{+}$will reduce $A$ either to $S^{ \pm}$or to a matrix of the form

$$
W_{w}^{ \pm}=\left(\begin{array}{cc}
0 & \pm 1 \\
\mp 1 & w
\end{array}\right), \quad w \equiv 0 \bmod 2 .
$$

By multiplying (4.5) from the right by $S^{ \pm}$, the resulting matrix will have $b=0$.

Suppose now that $|b|>1$. If $|a|<|b|$, we multiply $A$ from the right by $S^{ \pm}$, so that $|a|>|b|$; next we apply appropriately $T_{ \pm 2}^{+}$until $|a|<|b|$; we then apply $S^{ \pm}$and repeatedly multiply $A$ by $T_{ \pm 2}^{+}$until $|a|<|b|$, and so on. Since the elements of $A$ are integers, this procedure will stop after a finite number of steps when $a=0$. Then $A$ is of the form (4.5). 
The relations (4.3) can be verified straightforwardly by matrix multiplication. To complete the proof of theorem 4.1 we only have to check that no other relations independent of (4.3) exist, i.e. if $W=a_{1} a_{2} \cdots a_{k}=1_{2}$, where either $a_{j} \in G$ or $a_{j}^{-1} \in G$, then $W$ can be mapped into the void word by repeated applications of (4.3).

Let us consider the quotient group $\mathrm{P} \Gamma_{\theta}=\Gamma_{\theta} / \Lambda$, where $\Lambda=\left\{1_{2}, P\right\}$ is the centre of $\Gamma_{\theta}$ and the kernel of the homomorphism $\sigma: \Gamma_{\theta} \rightarrow \mathrm{P} \Gamma_{\theta}$. Let us denote

$$
S=\sigma\left(S^{ \pm}\right), \quad T_{2}=\sigma\left(T_{2}^{ \pm}\right) .
$$

Clearly $S$ and $T_{2}$ generate $\mathrm{P}_{\theta}$ and $\sigma$ maps the relations (4.3) into $S^{2}=1$. We now proceed in two steps: firstly, we prove that

$$
\mathrm{P} \Gamma_{\theta}=\left\langle S, T_{2} \mid S^{2}=1\right\rangle \text {. }
$$

Secondly, using (4.7), we show that (4.3) are the only relations in $\Gamma_{\theta}$.

Let us introduce the groups

$$
\Gamma(2)=\left\{A \in \mathrm{SL}(2, \mathbb{Z}) \mid A \equiv 1_{2} \bmod 2\right\} \quad \text { and } \quad \mathrm{P} \Gamma(2)=\Gamma(2) / \Lambda .
$$

It turns out (see, e.g. 116) that $\mathrm{P} \Gamma(2)$ is a free normal subgroup of $\mathrm{P} \Gamma_{\theta}$ of index two generated by

$$
T_{2} \text { and } \quad \bar{T}_{2}=S T_{2}^{-1} S .
$$

Furthermore, we have

$$
\mathrm{P} \Gamma_{\theta}=\mathrm{P} \Gamma(2)+S \mathrm{P} \Gamma(2) .
$$

Because of (4.8), proving (4.7) is equivalent to showing that

$$
\mathrm{P} \Gamma_{\theta}=\left\langle S, T_{2}, \bar{T}_{2} \mid S^{2}=1, S T_{2} S \bar{T}_{2}=1\right\rangle .
$$

Now, we have $\mathrm{P} \Gamma_{\theta}=\left\langle S, T_{2}, \bar{T}_{2}\right\rangle$. Therefore, by (4.9) each word

$$
W=a_{1} a_{2} \cdots a_{k}
$$

of the symbols $S^{\epsilon}, T_{2}^{\epsilon}$ and $\bar{T}_{2}^{\epsilon}, \epsilon= \pm 1$, can be mapped by a certain set of relations in $\mathrm{P} \Gamma_{\theta}$ into a string

$$
W^{\prime}=a_{1}^{\prime} a_{2}^{\prime} \cdots a_{r}^{\prime}
$$

where either $W^{\prime}$ is a sequence of the symbols $T_{2}^{\epsilon}$ and $\bar{T}_{2}^{\epsilon}$ or a word where $a_{1}^{\prime}=S^{\epsilon}$ and $a_{2}^{\prime} a_{3}^{\prime} \cdots a_{r}^{\prime}$ is a string that does not contain $S^{\epsilon}$. Since $\mathrm{P} \Gamma(2)$ is a free group, there are no relations between $T_{2}$ and $\bar{T}_{2}$, therefore (4.10) and (4.7) are a consequence of the following.

Lemma 4.3. The map $W \mapsto W^{\prime}$ is achieved by the relations

$$
S^{2}=1, \quad S T_{2} S \bar{T}_{2}=1 .
$$


Proof. Using $S^{2}=1$ any word $W$ can be transformed into a string where $S$ appears only with exponent one. Next, using both relations (4.13), all the sequences of the form

$$
\cdots S T_{2}^{\epsilon_{m} m} S \cdots, \quad \cdots S \bar{T}_{2}^{\epsilon_{n} n} S \cdots, \quad \epsilon_{m}, \epsilon_{n}= \pm 1, \quad m, n \in \mathbb{Z}^{+}
$$

can be turned into powers of $T_{2}$ and $\bar{T}_{2}$. Suppose now that only an even number of $S \mathrm{~s}$ are left into $W$. Then, we break $W$ into strings of the form

$$
\cdots S T_{2}^{\epsilon_{m} m} \bar{T}_{2}^{\epsilon_{n} n} \cdots T_{2}^{\epsilon_{p} p} S \cdots, \quad \epsilon_{m}, \epsilon_{m}, \epsilon_{p}= \pm 1, \quad m, n, p \in \mathbb{Z}^{+} .
$$

Such sequences can be transformed into powers of $T_{2}$ and $\bar{T}_{2}$ by inserting $S^{2}$ between each pair $T_{2}^{\epsilon_{m} m} \bar{T}_{2}^{\epsilon_{n} n}$. The resulting word $W^{\prime}$ does not contain $S$. Instead, if $W$ contains an odd number of $S \mathrm{~s}$, the transformation (4.15) will leave only one $S$ in $W$. Now, either $S$ is the first symbol, in which case we stop, or $W$ begins with a string of the type

$$
T_{2}^{\epsilon_{m} m} \bar{T}_{2}^{\epsilon_{n} n} \cdots T_{2}^{\epsilon_{p} p} S \cdots, \quad \epsilon_{m}, \epsilon_{n}, \epsilon_{p}= \pm 1, \quad m, n, p \in \mathbb{Z}^{+} .
$$

The symbol $S$ can then be moved in the first position by multiplying the word by $S^{2}$ from the left and by inserting $S^{2}$ between each pair $T_{2}^{\epsilon_{m} m} \bar{T}_{2}^{\epsilon_{n} n}$.

We now have left to show that no other relations except (4.3) exist among $S^{+}, P$ and $T_{2}^{+}$. Since $P$ commutes with both $S^{+}$and $T_{2}^{+}$, the only other possible relations must be of the form

$$
\begin{aligned}
& T_{2}^{+\epsilon_{m} m} P=1_{2}, \quad \epsilon_{m}= \pm 1, \quad m \in \mathbb{Z}^{+} \\
& P a_{1} a_{2} \cdots a_{k}=1_{2}, \\
& a_{1}^{\prime} a_{2}^{\prime} \cdots a_{r}^{\prime}=1_{2},
\end{aligned}
$$

where $a_{1} a_{2} \cdots a_{k}$ and $a_{1}^{\prime} a_{2}^{\prime} \cdots a_{k}^{\prime}$ are strings that contain both $S^{+}$and $T_{2}^{+}$but not $P$. Now, $T_{2}^{+}$is of infinite order; this excludes relation (4.16a) because, together with $P^{2}=1_{2}$, it would imply that $T_{2}^{+}$is of finite order. Relations of the type (4.160) and (4.160 are excluded because their images under the homomorphism $\sigma: \Gamma_{\theta} \rightarrow \mathrm{P}_{\theta}$ would be relations in $\mathrm{P}_{\theta}$ involving $S$ and $T_{2}$. This completes the proof of theorem 4.1.

\section{The choice of propagators}

Theorem 4.1 says that there exists a choice of phases in the definition of the propagators such that

$$
U_{N}(A B)=U_{N}(A) U_{N}(B), \quad A, B \in \Gamma_{\theta} .
$$

The quantum map $U_{N}(A)$ for arbitrary $A \in \Gamma_{\theta}$ is then given as a product of a certain finite sequence of $U_{N}\left(T_{ \pm 2}^{+}\right)$and $U_{N}\left(S^{ \pm}\right)$. Our goal is to prove that the propagators (2.3) are equivalent to such a product. This will be the aim of this section and section 6 .

Our strategy will be to prove that multiplicativity holds if any of the propagators (2.3) is multiplied either from the right or from the left by any of $U_{N}\left(T_{ \pm 2}^{+}\right)$or $U_{N}\left(S^{ \pm}\right)$. This will imply the equivalence of definitions (2.3) and (4.1), and will provide an independent proof of (5.1). 
It is a straightforward exercise to check that if we multiply any of the operators $(2.3 a),(2.3 b),(2.3 d)$ and (2.3d) from the left and from the right by $U_{N}\left(T_{ \pm 2}^{+}\right)$and $U_{N}\left(S^{ \pm}\right)$, then multiplicativity holds. Thus, it remains to be proved for the subset of $\Gamma_{\theta}$ such that $U_{N}(A)$ is of the form (2.3e), i.e.

$$
M=\left\{A \in \Gamma_{\theta} \mid a \neq 0, b \neq 0\right\} .
$$

The product of $A \in M$ with one of the generators of $\Gamma_{\theta}$ may not belong to $M$. However, it turns out that we need to check multiplicativity only if the product of $A$ with a given generator still belongs to $M$. For example, let us suppose that $A T_{m}^{ \pm}=S^{ \pm}$. Then we have

$$
U_{N}\left(A T_{m}^{ \pm}\right) U_{N}\left(T_{-m}^{ \pm}\right)=U_{N}\left(S^{ \pm}\right) U_{N}\left(T_{-m}^{ \pm}\right)=U_{N}(A)
$$

which implies

$$
U_{N}\left(A T_{m}^{ \pm}\right)=U_{N}(A) U_{N}\left(T_{m}^{ \pm}\right) .
$$

Moreover, it turns out that if multiplicativity holds if $A$ is multiplied from the right by the generators of $\Gamma_{\theta}$, then it also true when $A$ is multiplied from the left and vice versa. For example, let us consider $S^{ \pm}$. Since $\left(S^{ \pm} A\right)^{-1}=A^{-1} S^{\mp}$ and $U_{N}\left(A^{-1}\right)=U_{N}(A)^{-1}$, which can be verified directly from definitions (2.3), we obtain

$$
U_{N}\left(S^{ \pm} A\right)^{-1}=U_{N}\left(A^{-1} S^{\mp}\right)=U_{N}\left(A^{-1}\right) U_{N}\left(S^{\mp}\right)=\left(U_{N}\left(S^{ \pm}\right) U_{N}(A)\right)^{-1},
$$

which implies

$$
U_{N}\left(S^{ \pm} A\right)=U_{N}\left(S^{ \pm}\right) U_{N}(A) .
$$

An analogous argument applies to $T_{ \pm 2}^{+}$.

Proving multiplicativity for $T_{ \pm 2}^{+}$is quite straightforward. We need to show that

$$
U_{N}\left(T_{ \pm 2}^{+} A\right)=U_{N}\left(T_{ \pm 2}^{+}\right) U_{N}(A), \quad A, T_{ \pm 2}^{+} A \in M .
$$

We now apply both sides of the above equation to the Kronecker delta function $\delta_{0}(Q)$ (see appendix B for the definition of $\left.\delta_{\nu}(Q)\right)$ and evaluate their images at $Q=0$. Since the first row of $A$ is not affected by the multiplication by $T_{ \pm 2}^{+}$, we immediately obtain

$$
\left[U_{N}\left(T_{ \pm 2}^{+} A\right) \delta_{0}\right](0)=\sqrt{(b, N)} h(a, b) G\left(N_{b} a, b^{\prime}, 0\right)
$$

and

$$
\left[U_{N}\left(T_{ \pm 2}^{+}\right) U_{N}(A) \delta_{0}\right](0)=\sqrt{(b, N)} h(a, b) G\left(N_{b} a, b^{\prime}, 0\right)
$$

Since the propagators $U_{N}(A)$ form a projective representation of $\Gamma_{\theta}$, equations $(5.3)$ imply (5.2).

Proving multiplicativity when the generators are $S^{ \pm}$is more involved and will be dealt with in the next section. 


\section{Multiplication by $S^{ \pm}$}

We now show that

$$
U_{N}\left(A S^{ \pm}\right)=U_{N}(A) U_{N}\left(S^{ \pm}\right), \quad A, A S^{ \pm} \in M .
$$

We shall discuss the proof of (6.1) only for $S^{+}$, since

$$
A S^{-}=-A S^{+}, \quad A, A S^{-} \in M .
$$

Let us consider the two disjoint subsets

$$
\begin{aligned}
& K_{\mathrm{R}}=\left\{A \in M \mid A \equiv S^{+} \bmod 2, \quad A S^{+} \in M\right\}, \\
& L_{\mathrm{R}}=\left\{A \in M \mid A \equiv 1_{2} \bmod 2, \quad A S^{+} \in M\right\} .
\end{aligned}
$$

Clearly $K_{\mathrm{R}}=L_{\mathrm{R}} S^{+}$and $L_{\mathrm{R}}=K_{\mathrm{R}} S^{+}$. Thus, if (6.1) holds when $A \in K_{\mathrm{R}}$, it is also true if $A \in L_{\mathrm{R}}$. In fact, suppose that $A \in L_{\mathrm{R}}$, then we have $B=A S^{+} \in K_{\mathrm{R}}$ and

$$
U_{N}(A) U_{N}\left(S^{+}\right)=U_{N}\left(B S^{-}\right) U_{N}\left(S^{+}\right)=U_{N}(B)=U_{N}\left(A S^{+}\right) .
$$

Therefore, we need to prove (6.1) only for $A \in K_{\mathrm{R}}$.

As in the previous section, we shall check multiplicativity by applying both sides of equation (6.1) to $\delta_{\nu}(Q)$. The integer $\nu$ and the value of $Q$ at which the image of $\delta_{\nu}(Q)$ will be evaluated will be chosen appropriately in order to make the algebra simple.

We have three different cases to consider:

- $N_{a}$ is odd and $a^{\prime}$ is even;

- $N_{a}$ is even and $a^{\prime}$ is odd;

- $N_{a}$ and $a^{\prime}$ are both odd.

\subsection{Case 1: $N_{a}$ odd and $a^{\prime}$ even}

The appropriate function to use in this case is $\delta_{0}(Q)$. Let us first apply it to the left-hand side of (6.1). We easily obtain

$$
\left[U_{N}\left(A S^{+}\right) \delta_{0}\right](0)=\sqrt{(a, N)}\left(\frac{|a|}{|b|}\right)\left(\frac{\left|a^{\prime}\right|}{N_{a b}\left|b^{\prime}\right|}\right) \text { e }\left(\operatorname{sign}(a b)\left(|b|-N_{a b}\left|b^{\prime}\right|\right) / 8\right),
$$

where we have used $(b, N)^{2} \equiv 1 \bmod 8$.

The term $\left[U_{N}(A) U_{N}\left(S^{+}\right) \delta_{0}\right](0)$ needs some more work. The action of $U(A)$ on a vector $\Phi \in \mathcal{H}_{N}$ is given by

$$
\begin{aligned}
{\left[U_{N}(A) \Phi\right](Q) } & =\frac{1}{\sqrt{N}_{b}}\left(\frac{|a|}{|b|}\right)\left(\frac{N_{a b}\left|a^{\prime}\right|}{|b|^{\prime}}\right) \mathrm{e}\left(\operatorname{sign}(a b)\left(|b|-\left|b^{\prime}\right|\right) / 8\right) \\
& \times \sum_{\substack{Q^{\prime} \bmod N \\
(b, N) \mid\left(a Q^{\prime}-Q\right)}} \mathrm{e}\left(\frac{1}{2 N b}\left(d Q^{2}-2 Q Q^{\prime}+a Q^{\prime 2}\right)\right) \\
& \times \mathrm{e}\left(-\frac{a N_{b}\left(|a| N_{b} \backslash\left|b^{\prime}\right|\right)^{2}}{2 b^{\prime}}\left(\frac{a Q^{\prime}-Q}{(b, N)}\right)^{2}\right) \Phi\left(Q^{\prime}\right) .
\end{aligned}
$$


On the multiplicativity of quantum cat maps

The condition $a d-b c=1$ implies that $a$ and $b$ are mutually prime and that $d \equiv(a \backslash|b|) \bmod |b|$. Moreover, we have

$$
\begin{aligned}
& \left(a N_{b} \backslash\left|b^{\prime}\right|\right) \equiv d N_{b}^{\left(\phi\left(\left|b^{\prime}\right|\right)-1\right)} \bmod \left|b^{\prime}\right|, \\
& \left(b^{\prime} \backslash N_{b}\right) \equiv \frac{1-N_{b}^{2 \phi\left(\left|b^{\prime}\right|\right)}}{b^{\prime}} \equiv \frac{1-N_{b}^{\phi\left(\left|b^{\prime}\right|\right)}}{b^{\prime}} \bmod N_{b} .
\end{aligned}
$$

Using the above congruences and replacing $\Phi(Q)$ by $\left[U_{N}\left(S^{+}\right) \delta_{0}\right](Q)$, equation (6.3) becomes

$$
\begin{gathered}
{\left[U_{N}(A) U_{N}\left(S^{+}\right) \delta_{0}\right](0)=\frac{1}{\sqrt{N}_{b}}\left(\frac{|a|}{|b|}\right)\left(\frac{N_{a b}\left|a^{\prime}\right|}{\left|b^{\prime}\right|}\right) \mathrm{e}\left(\operatorname{sign}(a b)\left(|b|-\left|b^{\prime}\right|\right) / 8\right)} \\
\times \sum_{Q \bmod N_{b}} \mathrm{e}\left(\frac{1}{2 N_{a b}}\left(b^{\prime} \backslash N_{b}\right) a^{\prime} Q^{2}\right) .
\end{gathered}
$$

Since the above Gauss sum does not depend on the choice of the representative in the equivalence class of $\left(b^{\prime} \backslash N_{b}\right)$, we can choose $\operatorname{sign}\left[\left(b^{\prime} \backslash N_{b}\right)\right]=-\operatorname{sign}(b)$. This is also consistent with $(6.46)$. The sum in (6.5) now becomes

$$
\begin{gathered}
{\left[U_{N}(A) U_{N}\left(S^{+}\right) \delta_{0}\right](0)=\sqrt{(a, N)}\left(\frac{|a|}{|b|}\right)\left(\frac{N_{a b}\left|a^{\prime}\right|}{\left|b^{\prime}\right|}\right)\left(\frac{-\operatorname{sign}(b)\left|a^{\prime}\right|\left(b^{\prime} \backslash N_{b}\right)}{N_{a b}}\right)} \\
\times \mathrm{e}\left(\operatorname{sign}(a b)\left(|b|-\left|b^{\prime}\right|+N_{a b}-1\right) / 8\right) .
\end{gathered}
$$

The above equation is then transformed into (6.2) by using the equality

$$
\begin{aligned}
\left(\frac{N_{a b}\left|a^{\prime}\right|}{\left|b^{\prime}\right|}\right) & \left(\frac{-\operatorname{sign}(b)\left|a^{\prime}\right|\left(b^{\prime} \backslash N_{b}\right)}{N_{a b}}\right)= \\
& =\left(\frac{\left|a^{\prime}\right|}{N_{a b}\left|b^{\prime}\right|}\right) \mathrm{e}\left(-\operatorname{sign}(a b)\left(N_{a b}+N_{a b}\left|b^{\prime}\right|-\left|b^{\prime}\right|-1\right) / 8\right) .
\end{aligned}
$$

\subsection{Case 2: $N_{a}$ even and $a^{\prime}$ odd}

We shall follow the same technique as in section 6.1. The evaluation of $\left[U_{N}\left(A S^{+}\right) \delta_{0}\right](0)$ is straightforward:

$$
\left[U_{N}\left(A S^{+}\right) \delta_{0}\right](0)=\sqrt{(a, N)}\left(\frac{|a|}{|b|}\right)\left(\frac{N_{a b}\left|b^{\prime}\right|}{\left|a^{\prime}\right|}\right) \text { e }\left(\operatorname{sign}(a b)\left(|b|+\left|a^{\prime}\right|-1\right) / 8\right) \text {. }
$$

The computation of $\left[U_{N}(A) U_{N}\left(S^{+}\right) \delta_{0}\right](0)$ does not differ from the previous case until equation (6.6), which now becomes

$$
\begin{gathered}
{\left[U_{N}(A) U_{N}\left(S^{+}\right) \delta_{0}\right](0)=\sqrt{(a, N)}\left(\frac{|a|}{|b|}\right)\left(\frac{N_{a b}\left|a^{\prime}\right|}{\left|b^{\prime}\right|}\right)\left(\frac{N_{a b}}{-\operatorname{sign}(b)\left|a^{\prime}\right|\left(b^{\prime} \backslash N_{b}\right)}\right)} \\
\times \mathrm{e}\left(\operatorname{sign}(a b)\left(|b|-\left|b^{\prime}\right|\right) / 8+\left(b^{\prime} \backslash N_{b}\right) a^{\prime} / 8\right] .
\end{gathered}
$$

As in equation (6.6), we have chosen $\left(b^{\prime} \backslash N_{b}\right)$ so that $\operatorname{sign}\left[\left(b^{\prime} \backslash N_{b}\right)\right]=-\operatorname{sign}(b)$. Rearranging the Jacobi symbols gives

$$
\begin{gathered}
\left(\frac{N_{a b}\left|a^{\prime}\right|}{\left|b^{\prime}\right|}\right)\left(\frac{N_{a b}}{-\operatorname{sign}(b)\left|a^{\prime}\right|\left(b^{\prime} \backslash N_{b}\right)}\right)=\left(\frac{N_{a b}\left|b^{\prime}\right|}{\left|a^{\prime}\right|}\right)\left(\frac{N_{a b}}{-b^{\prime}\left(b^{\prime} \backslash N_{b}\right)}\right) \\
\times \mathrm{e}\left(-\operatorname{sign}(a b)\left(\left|a^{\prime}\right|\left|b^{\prime}\right|-\left|a^{\prime}\right|-\left|b^{\prime}\right|+1\right) / 8\right) .
\end{gathered}
$$


Finally, the equality of the right-hand sides of equations (6.7) and (6.8) follows from

$$
\left(\frac{N_{a b}}{-b^{\prime}\left(b^{\prime} \backslash N_{b}\right)}\right) \text { e }\left(a^{\prime}\left[\left(b^{\prime} \backslash N_{b}\right)-b^{\prime}\right] / 8\right)=1,
$$

which can be obtained directly from property (A.2) of the Jacobi symbol.

\subsection{Case 3: $N_{a}$ odd and $a^{\prime}$ odd}

If we chose $\delta_{0}(Q)$ in this case too, the sum (6.5) would be zero. Now the appropriate choice is $\frac{\delta_{(a, N)}}{2}(Q)$. We have

$$
\begin{aligned}
{\left[U_{N}\left(A S^{+}\right) \delta_{\frac{(a, N)}{2}}\right] } & (0)=\sqrt{(a, N)}\left(\frac{|a|}{|b|}\right)\left(\frac{N_{a b}\left|b^{\prime}\right|}{\left|a^{\prime}\right|}\right) \\
& \times \mathrm{e}\left(\operatorname{sign}(a b)\left(|b|+\left|a^{\prime}\right|-1\right) / 8\right) \mathrm{e}\left(-\frac{b^{\prime}}{8 N_{a b}}\left(a^{\prime} \backslash 8 N_{a b}\right)\right) .
\end{aligned}
$$

On the other hand, the computation of the right-hand side of equation (6.1) leads to

$$
\begin{aligned}
& {\left[U_{N}(A) U_{N}\left(S^{+}\right) \delta_{\frac{(a, N)}{2}}\right](0)=\sqrt{(a, N)}\left(\frac{|a|}{|b|}\right)\left(\frac{N_{a b}\left|a^{\prime}\right|}{\left|b^{\prime}\right|}\right)\left(\frac{-\operatorname{sign}(b)\left|a^{\prime}\right|\left(b^{\prime} \backslash N_{b}\right)}{N_{a b}}\right)} \\
& \quad \times \mathrm{e}\left(\operatorname{sign}(a b)\left(|b|-\left|b^{\prime}\right|+N_{a b}-1\right) / 8\right) \mathrm{e}\left(-\frac{1}{8 N_{a b}} b^{\prime}\left(a^{\prime} \backslash N_{a b}\right) 4^{2 \phi\left(N_{a b}\right)}\right) \cdot(6.1
\end{aligned}
$$

Using

$$
\begin{aligned}
\left(\frac{N_{a b}\left|a^{\prime}\right|}{\left|b^{\prime}\right|}\right) & \left(\frac{-\operatorname{sign}(b)\left|a^{\prime}\right|\left(b^{\prime} \backslash N_{b}\right)}{N_{a b}}\right)= \\
= & \left(\frac{N_{a b}\left|b^{\prime}\right|}{\left|a^{\prime}\right|}\right) \text { e }\left(\operatorname{sign}(a b)\left(\left|b^{\prime}\right|+\left|a^{\prime}\right|-N_{a b}\right) / 8-a^{\prime} b^{\prime} N_{a b} / 8\right),
\end{aligned}
$$

equation (6.10) simplifies to

$$
\begin{aligned}
& {\left[U_{N}(A) U_{N}\left(S^{+}\right) \delta_{\frac{(a, N)}{2}}\right](0)=\sqrt{(a, N)} \text { e }\left(-\frac{1}{8 N_{a b}} b^{\prime}\left(a^{\prime} \backslash N_{a b}\right) 4^{2 \phi\left(N_{a b}\right)}\right)} \\
& \quad \times\left(\frac{|a|}{|b|}\right)\left(\frac{N_{a b}\left|b^{\prime}\right|}{\left|a^{\prime}\right|}\right) \text { e }\left(\operatorname{sign}(a b)\left(|b|+\left|a^{\prime}\right|-1\right) / 8-a^{\prime} b^{\prime} N_{a b} / 8\right) .
\end{aligned}
$$

Since $\left(b^{\prime}, 8 N_{a b}\right)=1$, the equality of the right-hand sides of equations (6.9) and 6.11) follows from the congruence

$$
\left(a^{\prime} \backslash 8 N_{a b}\right) \equiv\left(a^{\prime} \backslash N_{a b}\right) 4^{2 \phi\left(N_{a b}\right)}+a^{\prime} N_{a b}^{2} \bmod 8 N_{a b},
$$

which can be easily proved using the Chinese remainder theorem.

The proof of theorem 2.1 is now completed. 


\section{Acknowledgments}

The author is grateful to David Farmer, Jon Keating, Pär Kurlberg, Jens Marklof and Zeév Rudnick for helpful discussions, and to Brian Conrey, the director of the American Institute of Mathematics, for kind hospitality while this work was completed and written.

\section{Appendix A. Some properties of the Jacobi symbol}

In this appendix we present the definitions and main properties of the Legendre and Jacobi symbols. For a more complete exposition the reader should consult a book on elementary Number Theory (see, e.g. [13]).

Let $p$ be a prime greater than 2 and $q \in \mathbb{Z}$. The Legendre symbol is defined by

$$
\left(\frac{q}{p}\right):= \begin{cases}1 & \text { if } \exists x \in \mathbb{Z} / p \mathbb{Z} \mid x^{2} \equiv q \bmod p \\ -1 & \text { if } \nexists x \in \mathbb{Z} / p \mathbb{Z} \mid x^{2} \equiv q \bmod p, \\ 0 & \text { if } p \mid q .\end{cases}
$$

The Legendre symbol has the following properties.

i.e.

$$
\begin{aligned}
& \left(\frac{q_{1} q_{2}}{p}\right)=\left(\frac{q_{1}}{p}\right)\left(\frac{q_{2}}{p}\right) \text { and }\left(\frac{q}{p_{1} p_{2}}\right)=\left(\frac{q}{p_{1}}\right)\left(\frac{q}{p_{2}}\right), \\
& \left(\frac{1}{p}\right)=1 \text { and }\left(\frac{q+p}{p}\right)=\left(\frac{q}{p}\right), \\
& \left(\frac{-1}{p}\right)= \begin{cases}1 & \text { for } p \equiv 1 \bmod 4 \\
-1 & \text { for } p \equiv 3 \bmod 4,\end{cases}
\end{aligned}
$$

$$
\left(\frac{-1}{p}\right)=\mathrm{e}( \pm(p-1) / 4)
$$

$$
\left(\frac{2}{p}\right)= \begin{cases}1 & \text { for } p \equiv \pm 1 \bmod 8 \\ -1 & \text { for } p \equiv \pm 3 \bmod 8\end{cases}
$$

which can be written

$$
\left(\frac{2}{p}\right)=\mathrm{e}\left( \pm\left(p^{2}-1\right) / 16\right)
$$

(v) if $p$ and $q$ are both prime and greater than 2 , then

$$
\left(\frac{p}{q}\right)\left(\frac{q}{p}\right)= \begin{cases}-1 & \text { if } p \equiv q \equiv 3 \\ 1 & \text { otherwise }\end{cases}
$$

or equivalently

$$
\left.\left(\frac{p}{q}\right)\left(\frac{q}{p}\right)=\mathrm{e}( \pm(p-1)(q-1)) / 8\right) .
$$


Property (v) is known as the quadratic reciprocity law. The sign in the arguments of the above exponentials is obviously arbitrary. We make extensive use of this simple fact in this paper.

Let $r$ be an odd positive integer and $q \in \mathbb{Z}$. Let $r=p_{1} p_{2} \cdots p_{n}$ where the $p_{i}$ are (not necessarily distinct) primes. The symbol

$$
\left(\frac{q}{r}\right):=\left(\frac{q}{p_{1}}\right)\left(\frac{q}{p_{2}}\right) \cdots\left(\frac{q}{p_{n}}\right)
$$

is called the Jacobi symbol.

Properties (i) and (ii) trivially extend to the Jacobi symbol. Also (iii-v) apply to the Jacobi symbol (for reciprocity both numbers must be odd and positive). This follows from the congruences

$$
\begin{aligned}
& \sum_{k=1}^{n} \frac{r_{k}-1}{2} \equiv \frac{r_{1} r_{2} \cdots r_{n}-1}{2} \bmod 2 \\
& \sum_{k=1}^{n} \frac{r_{k}^{2}-1}{8} \equiv \frac{r_{1}^{2} r_{2}^{2} \cdots r_{n}^{2}-1}{8} \bmod 2
\end{aligned}
$$

where $r_{1}, r_{2}, \ldots, r_{n}$ are odd integers.

Let $q$ and $r$ be positive odd integers and $r \equiv \pm 1 \bmod 4 q$. Using the reciprocity law and properties (ii) and (iii) one easily obtains

$$
\left(\frac{q}{r}\right)=1 \text {. }
$$

Now, let $q=2^{\alpha} \bar{q}$, with $\alpha \geq 1$ and $\bar{q}$ odd, and let $r \equiv \pm 1 \bmod 4 \bar{q}$. Then, by (A.1) and using (i) and (iv), we have

$$
\left(\frac{q}{r}\right)= \begin{cases}-1 & \text { if } q \equiv 2 \bmod 4 \text { and } r \equiv \pm 3 \bmod 8 \\ 1 & \text { otherwise }\end{cases}
$$

\section{Appendix B. Quantum mechanics on the two-torus}

We briefly review the quantum mechanics of systems whose classical phase space is $\mathbb{T}^{2}$. For more details see, e.g. 112, 8, 4, 3, 10. Without loss of generality, we restrict to the case when $\boldsymbol{\varphi}=(0,0)$, i.e. the wavefunction is exactly periodic in both the position and momentum bases.

The periodicity of the wavefunction in both representations has two important

consequences. Firstly, both $\psi(q)$ and $\hat{\psi}(p)$ are superpositions of delta functions supported on the lattices points $q=2 \pi \hbar Q$ and $p=2 \pi \hbar P$ respectively, where $Q, P \in \mathbb{Z}$. That is,

$$
\psi(q)=\sum_{m \in \mathbb{Z}} \sum_{Q=0}^{N-1} \Psi(Q) \delta\left(q-\frac{Q}{N}+m\right)
$$


with $\Psi(Q+N)=\Psi(Q)$. Secondly, $2 \pi \hbar$ must be an inverse integer, i.e. $N=1 / 2 \pi \hbar$. It follows that the Hilbert space may be identified with the $N$-dimensional vector space $\mathcal{H}_{N}=\mathrm{L}^{2}(\mathbb{Z} / N \mathbb{Z}) \cong \mathbb{C}^{N}$. For $\nu=0, \ldots, N-1$ the set of functions

$$
\delta_{\nu}(Q):= \begin{cases}\sqrt{N} & \text { if } Q \equiv \nu \bmod N, \\ 0 & \text { if } Q \neq \equiv \nu \bmod N\end{cases}
$$

forms an orthogonal basis in $\mathcal{H}_{N}$.

Quantization means mapping of observables $f \in C^{\infty}\left(\mathbb{T}^{2}\right)$ into operators $\mathrm{Op}_{N}(f)$ acting on $\mathcal{H}_{N}$ such that as $N \rightarrow \infty$, i.e. $\hbar \rightarrow 0, \mathrm{Op}_{N}(f)$ tends to $f$. More precisely, if $f, g \in C^{\infty}\left(\mathbb{T}^{2}\right)$ then we require that

$$
\frac{N}{2 \pi}\left[\mathrm{Op}_{N}(f), \mathrm{Op}_{N}(g)\right]-\mathrm{Op}_{N}(\{f, g\}) \underset{N \rightarrow \infty}{\longrightarrow} 0 .
$$

Here $[\cdot, \cdot]$ denotes the commutator of two operators and

$$
\{f, g\}=\frac{\partial f}{\partial p} \frac{\partial g}{\partial q}-\frac{\partial f}{\partial q} \frac{\partial g}{\partial p}
$$

are the Poisson brackets of two observables.

In order to define a quantization satisfying (B.2), we need to introduce the translation operators

$$
\begin{aligned}
& t_{1} \Phi(Q):=\Phi(Q) \mathrm{e}\left(\frac{Q}{N}\right), \\
& t_{2} \Phi(Q):=\Phi(Q+1),
\end{aligned}
$$

which may be thought of as the analogue of $\mathrm{e}(\hat{q})$ and $\mathrm{e}(\hat{p})$, where $\hat{q}$ and $\hat{p}$ are the usual position and momentum operators in $\mathrm{L}^{2}(\mathbb{R})$. For any $m, n \in \mathbb{Z}$, we have the following commutation relation

$$
t_{1}^{m} t_{2}^{n}=\mathrm{e}\left(-\frac{m n}{N}\right) t_{2}^{n} t_{1}^{m}
$$

Note that $t_{1}^{N}=t_{2}^{N}=1_{N}$.

The Weyl-Heisenberg operators are defined by

$$
T_{N}(\boldsymbol{n}):=\mathrm{e}\left(-\frac{n_{1} n_{2}}{2 N}\right) t_{2}^{n_{2}} t_{1}^{n_{1}}
$$

where $\boldsymbol{n}=\left(n_{1}, n_{2}\right)$. We then have the following multiplication rule

$$
T_{N}(\boldsymbol{m}) T_{N}(\boldsymbol{n})=\mathrm{e}\left(-\frac{1}{2 N} \omega(\boldsymbol{m}, \boldsymbol{n})\right) T_{N}(\boldsymbol{m}+\boldsymbol{n}),
$$

where $\omega(\boldsymbol{m}, \boldsymbol{n}):=m_{1} n_{2}-m_{2} n_{1}$ is the standard symplectic form.

Let $f \in C^{\infty}\left(\mathbb{T}^{2}\right)$ be a classical observable on $\mathbb{T}^{2}$ whose Fourier series is given by

$$
f(\boldsymbol{z})=\sum_{\boldsymbol{m} \in \mathbb{Z}^{2}} \hat{f}_{\boldsymbol{m}} \mathrm{e}(\boldsymbol{z} \cdot \boldsymbol{m}), \quad \boldsymbol{z}=\left(\begin{array}{c}
q \\
p
\end{array}\right) \in \mathbb{T}^{2} .
$$

The Weyl quantization of $f$ is defined as

$$
\mathrm{Op}_{N}(f):=\sum_{\boldsymbol{m} \in \mathbb{Z}^{2}} \hat{f}_{\boldsymbol{m}} T_{N}(\boldsymbol{m}) .
$$

If $f$ is real, then $\mathrm{Op}_{N}(f)$ is self-adjoint. Moreover, it can be shown that $\mathrm{Op}_{N}(f)$ satisfies (B.2). 


\section{Appendix C. Proof of equation (2.8)}

In this appendix we show that

$$
h(a, b)=h(d, b), \quad A=\left(\begin{array}{ll}
a & b \\
c & d
\end{array}\right) \in \mathrm{SL}(2, \mathbb{Z}),
$$

where the function $h$ is defined in (2.4). If $a$ is even we have

$$
h(a, b)=\left(\frac{\operatorname{sign}(a d)}{|b|}\right)\left(\frac{|d|}{|b|}\right) \text { e }(\operatorname{sign}(a b)(|b|-1) / 8) .
$$

If $\operatorname{sign}(a d)=1$ then $\operatorname{sign}(a b)=\operatorname{sign}(b d)$ and (2.8) follows immediately. If $\operatorname{sign}(a d)=-1$ then

$$
\left(\frac{-1}{|b|}\right) \mathrm{e}(\operatorname{sign}(a b)(|b|-1) / 8)=\mathrm{e}(-\operatorname{sign}(a b)(|b|-1) / 8)=\mathrm{e}(\operatorname{sign}(b d)(|b|-1) / 8),
$$

which proves (2.8).

Now, let $a$ be an odd number and $b=2^{\alpha} \bar{b}$, where $\alpha \geq 1$ and $\bar{b}$ is odd. The condition $a d-b c=1$ implies that $|a d| \equiv \pm 1 \bmod 4|\bar{b}|$. Moreover, we have either

$$
a \equiv d \bmod 8
$$

or

$$
d \equiv a+4 \bmod 8 \text {. }
$$

The congruence (C.1) implies

$$
h(a, b)=\left(\frac{|b|}{|a d|}\right)\left(\frac{|b|}{|d|}\right) \text { e }(-\operatorname{sign}(a b)|a| / 8)=h(d, b),
$$

which follows from property (A.2) of the Jacobi symbol and from the equivalence $a d \equiv 1 \bmod 8$. If (C.2) holds, then $a d \equiv-3 \bmod 8$ and $b=2 \bar{b}$. Therefore, we have

$$
\left(\frac{|b|}{|a d|}\right)=\left(\frac{2}{|a d|}\right)\left(\frac{|\bar{b}|}{|a d|}\right)=-1 \text {. }
$$

Combining (C.2) and (C.3) we obtain

$$
h(a, b)=\left(\frac{|b|}{|d|}\right) \mathrm{e}(-\operatorname{sign}(a b)(|a|+4) / 8)=h(d, b) .
$$

\section{References}

[1] Balian R and Itzykson C 1986 Observations sur la mécanique quantique finie C. R. Acad. Sc. Paris $303773-8$

[2] Beyl F R 1986 The Schur multiplicator of $\operatorname{SL}(2, \mathbb{Z} / m \mathbb{Z})$ and the congruence subgroup property Math. Zeit. 191 23-42

[3] De Bièvre S, Degli Esposti M and Giachetti R 1996 Quantization of a class of piecewise affine transformations on the torus Commun. Math. Phys. 176 73-94

[4] Degli Esposti M 1993 Quantization of the orientation preserving automorphisms of the torus Ann. Inst. H. Poincaré $58323-41$ 
[5] Gelbart S 1976 Weil's representation and the spectrum of the metaplectic group Lectures Notes in Mathematics 530 (Berlin: Springer-Verlag)

[6] Keating J P and Mezzadri F 2000 Pseudo-symmetries of Anosov maps and spectral statistics Nonlinearity 13 747-75

[7] Keating J P, Mezzadri F and Robbins J M 1999 Quantum boundary conditions for torus maps Nonlinearity 12 579-91

[8] Knabe S 1990 On the quantization of Arnold's cat J. Phys. A: Math. Gen. 23 2013-25

[9] Kubota $\mathrm{T} 1969$ On automorphic functions and the reciprocity law in a number field Lectures in Mathematics (Tokyo: Department of Mathematics, Kyoto University, No. 2 Kinokuniya BookStore Co. Ltd.)

[10] Kurlberg P and Rudnick Z 2000 Hecke theory and equidistribution for the quantization of linear maps of the torus Duke Math. J. 103 47-78

[11] Kurlberg P and Rudnick Z 2001 Value distribution for eigenfunctions of desymmetrized quantum maps Internat. Math. Res. Notices 18 995-1002

[12] Hannay J H and Berry M V 1980 Quantization of linear maps on the torus - Fresnel diffraction by a periodic grating Physica D 1 267-90

[13] Ireland K and Rosen M 1990 A Classical Introduction to Modern Number Theory (New York: Springer-Verlag)

[14] Mennicke J 1967 On Ihara's modular group Invent. Math. 4 202-28

[15] Nobs A 1976 Die irreduziblen Darstellungen der Gruppen $\mathrm{SL}_{2}\left(\mathbb{Z}_{p}\right)$, insbesondere $\mathrm{SL}_{2}\left(\mathbb{Z}_{2}\right)$ I. Comment. Math. Helvetici $\mathbf{5 1}$ 465-89

[16] Rankin R A 1976 Modular forms and functions (Cambridge: Cambridge University Press)

[17] Shimura G 1971 Introduction to the arithmetic theory of automorphic functions (Princeton: Princeton University Press and Iwanami Shoten, Publishers)

[18] Schur I 1907 Untersuchungen über die Darstellung der endlichen Gruppen durch gebrochene lineare Substitutionen J. Reine Angew. Math. 132 85-137

[19] Zelditch S 1997 Index and dynamics of quantized contact transformations Ann. Inst. Fourier 47 $305-65$ 